\title{
Aspectos epidemiológicos del control antimalarico en un país libre de infecciones autóctonas, Chile.
}

\section{Epidemiological aspects of antimalarial control in a country free of autochthonous infections, Chile.}

\author{
Rubén Mercado $\mathrm{P}^{1}{ }^{\mathbb{D}}$, Sebastián Peña $\mathrm{F}^{2}$, Renzo Tassara $\mathrm{O}^{3}$, Edurne Urarte $\mathrm{I}^{4}$, Marisol Denegri $\mathrm{C}^{5}$.
}

\begin{abstract}
RESUMEN
Objetivo. Realizar analisis bibliográfico referente a malaria importada en Chile. Materiales y métodos. Se revisaron publicaciones periódicas, informes y boletines epidemiológicos publicados en Chile entre 1945 y 2020. Los casos se tabularon en una base de datos en términos de número de casos, género, nacionalidad y/o procedencia. Datos de presencia del vector se recopilaron en términos de localidad de muestreo. Resultados. Veinticuatro casos de malaria importados (13 chilenos) al territorio se notificaron en el periodo. Entre 1990 y 2001 hubo 66 nuevos casos, hombres (75,8\%). Entre 2011 y 2019 se comunicaron 114 nuevos casos,uno de ellos mortal, (único fallecimiento desde el año 2005). En el rango de edad de 25-45 años se concentra la mayoría de los infectados. En forma continua, se ha detectado mayoritariamente criaderos naturales de mosquitos Anopheles pseudopuntipennis en zona norte del país. Conclusiones. El aumento de
\end{abstract}

los viajes internacionales de chilenos y de la tasa de inmigración de extranjeros podría haber aumentado la notificación. La ausencia de casos autóctonos se habría logrado por el diagnóstico precoz, aislamiento, tratamiento y eliminación de focos de crianza de Anopheles. Desde el 2004 malaria es enfermedad de notificación obligatoria inmediata en Chile.

Palabras claves: malaria, paludismo, Plasmodium, Anopheles, epidemiologia.
Citación (Vancouver): Aspectos epidemiológicos del control anti malarico en un país libre de infecciones autóctonas, Chile. :30-31 . doi: 10.21897/25394622.2522

\footnotetext{
PhD, Prof. Asociado, Unidad Docente de Parasitología, Departamento de Cirugía y Pediatría Occidente, Facultad de Medicina, Universidad de Chile. Santiago, Chile. rubenmercado@uchile.cl

${ }^{2} \mathrm{MsC}$, Inst. Adjunto, Unidad Docente de Parasitología, Departamento de Cirugía y Pediatría Occidente, Facultad de Medicina, Universidad de Chile. Santiago, Chile. sebastianpena@uchile.cl

${ }^{3} \mathrm{MsC}$, Prof. Asociado, Unidad Docente de Parasitología, Departamento de Cirugía y Pediatría Occidente, Facultad de Medicina, Universidad de Chile. Santiago, Chile. rtassara@med.uchile.cl

${ }^{4}$ Lic., Prof. Asociado, Unidad Docente de Parasitología, Departamento de Cirugía y Pediatría Occidente, Facultad de Medicina, Universidad de Chile. Santiago, Chile. edurneur@gmail.com

${ }^{5}$ Lic., Prof. Asistente, Unidad Docente de Parasitología, Departamento de Cirugía y Pediatría Occidente, Facultad de Medicina, Universidad de Chile. Santiago, Chile. marisoldenegri@hotmail.com
} 


\begin{abstract}
Objective. To carry out bibliographic analysis regarding imported malaria in Chile. Materials and methods. Periodic publications, reports, and epidemiological bulletins published in Chile between 1945 and 2020 were reviewed. The cases were tabulated in a database in terms of the number of cases, gender, nationality, and/or origin. Vector presence data were collected in terms of sampling location. Results. Twenty-four imported malaria cases (13 Chileans) to the territory were reported in the period. Between 1990 and 2001 there were 66 new cases, men (75.8\%). Between 2011 and 2019, 114 new cases were reported, one of them fatal, (the only death since 2005). Most of those infected are concentrated in the 25-45 year age range. Continuously, most natural breeding sites for Anopheles pseudopuntipennis mosquitoes have been detected in the northern part of the country. Conclusions. The increase in international travel by Chileans and the immigration rate of foreigners could have increased notification. The absence of autochthonous cases would have been achieved by the early diagnosis, isolation, treatment, and elimination of breeding foci of Anopheles. Since 2004, malaria has been a disease of immediate mandatory notification in Chile.
\end{abstract}

Key words: malaria, paludism, Plasmodium, Anopheles, epidemiology.

\section{REFERENCIAS}

1. MinisteriodeSaluddeChile(2020).Informe: situación epidemiológica, diagnóstico y tratamiento de malaria. Acceso 2603-2021. https://diprece.minsal.cl/ wp-content/uploads/2021/02/InformeSituacion-epidemiologica-diagnosticoy-tratamiento-de-malaria-2020.pdf

2. Instituto de Salud Pública de Chile. (2012). Boletín ISP. Vigilancia de Malaria. Chile, 2005-2012. Acceso 2603-2021. https://www.ispch.cl/sites/ default/files/boletin malaria 0.pdf

3. Schenone F, H., Olea N, A., Rojas S, A., \& García D, N. (2002). Malaria en Chile: 1913 - 2001. Revista Médica de Chile, 130(10), 1170-1176. https://doi. org/10.4067/s0034-98872002001000013 